\title{
Study on Transfer Alignment Stable Targeting Method of Moving Base Based on Velocity Plus Attitude Matching Algorithm
}

\author{
Zhen Wang ${ }^{1,}$, Min $\mathrm{Gao}^{1}$, Fengqi Gao ${ }^{1}$ and Weiwei Gao ${ }^{1}$ \\ ${ }^{1}$ Shijiazhuang Mechanical Engineering College, Shijiazhuang 050003, China \\ awangzh822@sina.com
}

Keywords: transfer alignment; stable targeting; velocity plus attitude matching; Kalman filter.

\begin{abstract}
In the trend of the mobile warfare, the researching moving base alignment method of vehicle mounted missile is very valuable.A new stable targeting method based on the transfer alignment of speed and attitude matching is proposed to solve the problem of a longer time alignment and a greater impact by the device drift for single inertial measurement unit(IMU) system. Simulation results show that the initial alignment can be implemented within 9 seconds for optoelectronic device's IMU, and platform misalignment angle can be estimated accurately. The results for the attitude angle compensation can greatly improve the accuracy of stable targeting.
\end{abstract}

\section{Introduction}

The requirements of modern war on the reaction speed of a missile and hitting accuracy are more and more high. Before entering the navigation work, missile's inertial navigation system must have a initial alignment. Typically, the missile was launched from the carrier,so so the rapid and accurate initial alignment of the inertial navigation system in the carrier is becoming more and more important. Initial alignment, in general is to determine the attitude relationship between the carrier coordinate system and navigation coordinate system[1].

Stable targeting is such a process, in the traveling process of chariots, by measuring the chariot photoelectric aiming equipment relative attitude change of geodetic coordinate system after the orientation of target, it has an attitude compensation, so that the aiming equipment always aim at the target. Among them, in the geodetic coordinate system for photoelectric aiming equipment accurate positioning change of attitude angle is the key technology to realize the stabilization of targeting. Some land chariot equipment on the target aimed shot must be carried out in a static base or having a low precision. The main reasons are as follows: 1 ) Photoelectric device's IMU needs initial alignment before work, single IMU only in the static base can achieve the precise alignment, and alignment time is long.2) In the process of single IMU marching with time variation of each parameter may diverge,it's unable to accurately locate the attitude angle changing of aiming equipment[2]. In the trend of the mobile warfare, the researching moving base alignment method of vehicle mounted missile is very valuable.

The method of transfer alignment on moving base is the comprehensive utilization of the chariot master inertial navigation information and the information of sins on optoelectronic device. It uses Calman filter accurately estimate the change of attitude angle, which is photoelectric equipment relative to earth coordinates. Because the main INS is with high precision, in moving base condition, it can make rapid initial alignment on the photoelectric equipment's sins; The fusion of master inertial navigation information and sub inertial navigation information can estimate the parameters errors, which can realize error compensation of attitude measurement system[3]. This not only avoids the initial alignment of the static base condition, and can rapidly improve the precision of stable targeting. 


\section{Overall Scheme}

Transfer alignment method consists of measurement parameter matching method and calculation parameter matching method. The former mainly includes acceleration matching and angular rate matching, the latter mainly includes position and velocity matching[4]. In practice, people often use the combination parameter matching method, in which the velocity plus attitude matching method because of its high alignment precision and speed is especially widely used[5,6]. Taking the velocity plus attitude matching method as the basis, we use the transfer alignment on moving base. The system's overall scheme is shown in Fig. 1.

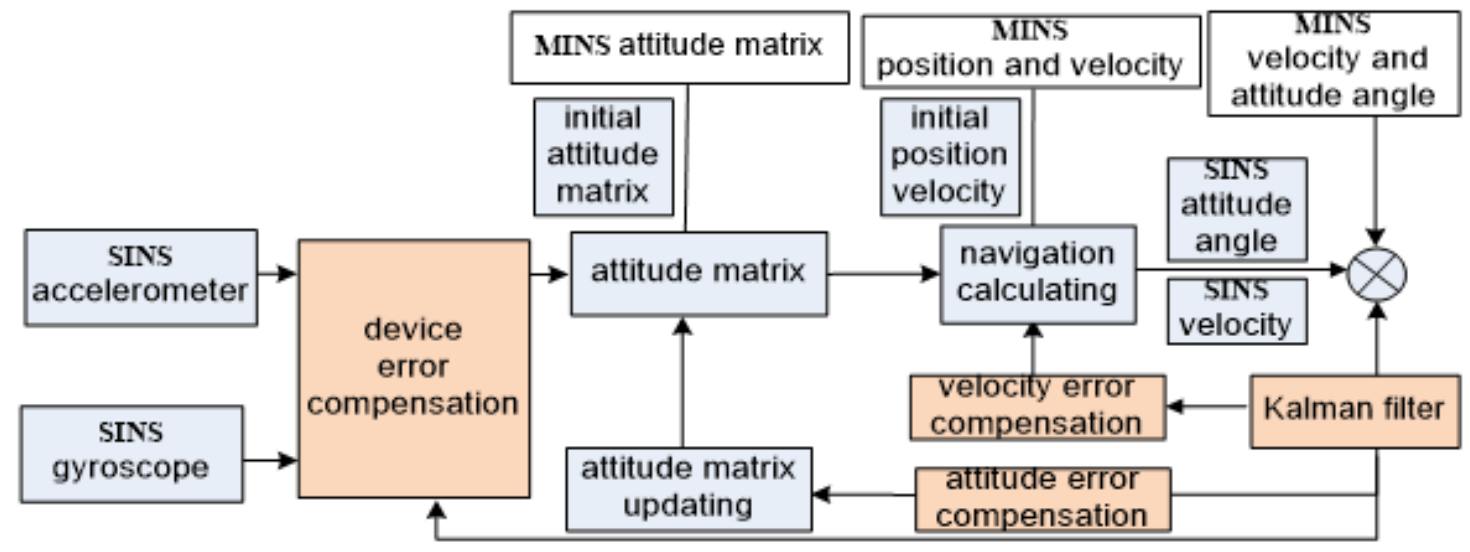

Fig. 1 The overall scheme of velocity plus attitude matching transfer alignment

We use speed, position, attitude information of chariot main inertial MINS to initialize sub inertial navigation SINS on photoelectric device, instead of SINS initial alignment on stationary base. The information of velocity, attitude angle,which is compared from SINS and MINS,is campared. The comparison results are as the Calman filter input. Calman filter makes an optimal estimation of state variables,such as attitude error, velocity error, device error. Through error compensation, we get the exact parameter information. Finally, we get three attitude angles of SINS relative to earth coordinates. By the attitude angle compensation, the objective of chariot's stable targeting was achieved.

\section{Mathematical Model}

Equation of State For Transfer Alignment. The section headings are in boldface capital and lowercase letters. Second level headings are typed as part of the succeeding paragraph (like the subsection heading of this paragraph). In transfer alignment, because the master inertial navigation system precision than the sub inertial navigation system is several orders of magnitude higher, the master INS is as a reference, the calculated navigation coordinate system can be considered true navigation coordinate system[7]. Navigation coordinate $n$ is regarded as the East - North - Up geography coordinate system. $\mathrm{n}^{\prime}$ represents the SINS navigation coordinate calculation. i represents the inertial coordinate system. ${ }^{b}$ represents the carrier coordinates. The error state equation is derived as follows:

First, velocity error equations.

Inertial navigation basic equation, $\dot{\mathrm{V}}^{\mathrm{n}}=f^{n}-2\left(\omega_{i e}+\omega_{e n}\right) \times V^{n}+g^{n}$

In the formula, $\omega_{i e}$ is the earth rotation angular velocity, $\omega_{e n}$ is navigation system angular velocity relative to the earth. $f^{n}$ is a projection of the accelerometer output is in the navigation system. $V^{n}$ is navigation system of the line speed. ${ }^{g^{n}}$ is a projection of acceleration of gravity in the navigation system.

By using the variational method, we calculate the formula, 


$$
\delta \dot{\mathrm{V}}^{\mathrm{n}}=\delta f^{n}-2\left(\delta \omega_{i e}+\delta \omega_{e n}\right) \times V^{n}-2\left(\omega_{i e}+\omega_{e n}\right) \times \delta V^{n}+\delta g^{n}
$$

$\varphi^{\mathrm{n}}$ stands for the sub inertial navigation computing platform misalignment angles. $f^{n^{\prime}}$ is the actual accelerometer output. The projection of accelerometer measurement error in navigation system is $\nabla^{n}, \delta g^{n}=0$ (As the chariot moves on the ground, the errors can be ignored). Formula of (3) is brought into the formula of (2). We get the formula of (4).

$$
\begin{aligned}
& \delta f^{n}=f^{n^{\prime}}-f^{n} \\
& \delta \dot{V}^{\mathrm{n}}=f^{n} \times \varphi^{\mathrm{n}}-2\left(\delta \omega_{i e}+\delta \omega_{e n}\right) \times V^{n}-2\left(\omega_{i e}+\omega_{e n}\right) \times \delta V^{n}+\nabla^{n}
\end{aligned}
$$

Then,the attitude error equation.

The attitude calculation matrix of sub inertial navigation is $C_{b}^{n^{\prime}}$, the attitude calculation matrix of main inertial navigation is $C_{b}^{n}$. Their relationships are as follows.

$C_{b}^{n^{\prime}}=C_{n}^{n^{\prime}} C_{b}^{n}$

In the formula (5), $C_{n}^{n^{\prime}}$ is a mathematical strapdown platform alignment error. In the small misalignment angles case, $C_{n}^{n^{\prime}}$ can be expressed as formula (6)

$$
C_{n}^{n^{\prime}}=I-\varphi^{n} \times=\left[\begin{array}{ccc}
1 & \varphi_{z} & -\varphi_{y} \\
-\varphi_{z} & 1 & \varphi_{x} \\
\varphi_{y} & -\varphi_{x} & 1
\end{array}\right]
$$

In the formula (6), $\varphi_{x}, \varphi_{y}, \varphi_{z}$ respectively stand as three misalignment angles in directions of north, East,Up.

$$
\omega_{i n^{\prime}}^{n^{\prime}}=C_{n}^{n^{\prime}} \omega_{i n}^{n}+\dot{\varphi}^{n}=\omega_{i n}^{n}-\varphi^{n} \times \omega_{i n}^{n}+\dot{\varphi}^{n}
$$

We write a formula $\delta \omega_{i n}^{n}=\delta \omega_{i e}^{n}+\delta \omega_{e n}^{n}, \varepsilon^{n}$ is the equivalent gyro drift in the navigation system. After simplification, omitting the two order terms, we get the attitude error formula (8).

$$
\dot{\varphi}^{n}=-\omega_{i n}^{n} \times \varphi^{n}+\delta \omega_{i n}^{n}-\varepsilon^{n}
$$

Here is device error equation.

The main error of gyro and accelerometer can be divided into constant error and random walk error. We set the symbols as follows, ${ }^{\varepsilon_{d}}$ is gyro constant error, ${ }^{\varepsilon_{w}}$ is gyro andom walk error, $\nabla_{d}$ is the constant error of accelerometer, $\nabla_{w}$ is andom walk error of accelerometer. $w_{\nabla}, w_{\varepsilon}$ represent different standard deviation of white noise. The accelerometer and gyro error equations are as follows.

$$
\begin{aligned}
& \dot{\nabla}=\dot{\nabla}_{d}+\dot{\nabla}_{w}=w_{\nabla} \\
& \dot{\varepsilon}=\dot{\varepsilon}_{d}+\dot{\varepsilon}_{w}=w_{\varepsilon}
\end{aligned}
$$

Integrating the above error equation, we get the state equations of transfer alignment.

$$
\dot{X}=F X+W
$$

Due to the chariot as the research object, in the establishment of the state equation, we think that the vertical channel speed is always zero, we don't consider the effect of vertical channel. $L$ stands for the geographic latitude, where is the carrier in. $\lambda$ represents the longitude. $h$ represents the height, $R_{e}$ stands for the earth radius $6378245 \mathrm{~m}, e$ represents the earth ellipticity $1 / 298.3 . R_{E}=R_{e} /\left(1-e \sin ^{2} L\right)$, this represents the local prime vertical plane curvature radius. $R_{N}=R_{e} /\left(1+2 e-3 e \sin ^{2} L\right)$, this indicates that the main curvature radius, it with the prime plane vertical. $C_{b}^{n}=\left(T_{i j}\right)_{3 \times 3}$, Each part of equation of state are as follows:

$$
X=\left[\begin{array}{llllllllll}
\delta V_{x}^{n} & \delta V_{y}^{n} & \varphi_{x}^{n} & \varphi_{y}^{n} & \varphi_{z}^{n} & \nabla_{x} & \nabla_{y} & \varepsilon_{x} & \varepsilon_{y} & \varepsilon_{z}
\end{array}\right]^{T}
$$




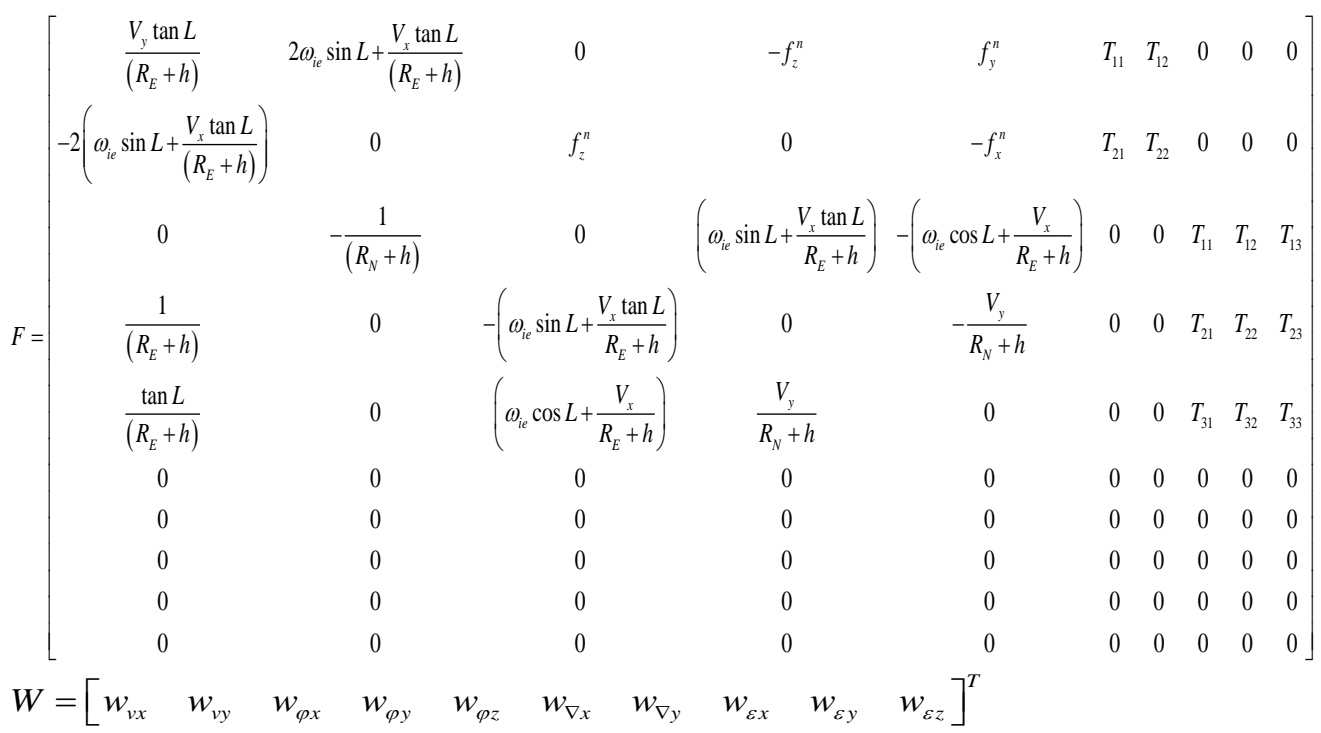

In $W$, during the simulation, each element can be regarded as zero mean white noise.

The Measurement Equation of Transfer Alignment. $\delta V_{x}^{n}, \delta V_{y}^{n}$ represent the main, sub ins level speed difference, $\delta \gamma, \delta \theta, \delta \psi$ represent the roll angle, pitch angle and yaw angle difference. Transfer alignment measurement equation as follows: $\boldsymbol{Z}=\boldsymbol{H} \boldsymbol{X}+\boldsymbol{V}$

In the formula, we use the measured element representation formula.

$$
Z=\left[\begin{array}{lllll}
\delta V_{x}^{n} & \delta V_{y}^{n} & \delta \gamma & \delta \theta & \delta \psi
\end{array}\right]^{T}
$$

The observation matrix, $H=\left[\begin{array}{cccccccccc}1 & 0 & 0 & 0 & 0 & 0 & 0 & 0 & 0 & 0 \\ 0 & 1 & 0 & 0 & 0 & 0 & 0 & 0 & 0 & 0 \\ 0 & 0 & \frac{-T_{22}}{\sqrt{1-T_{32}^{2}}} & \frac{T_{12}}{\sqrt{1-T_{32}^{2}}} & 0 & 0 & 0 & 0 & 0 & 0 \\ 0 & 0 & \frac{T_{21} T_{33}-T_{31} T_{23}}{T_{31}^{2}+T_{33}^{2}} & \frac{T_{13} T_{31}-T_{113} T_{33}}{T_{31}^{2}+T_{33}^{2}} & 0 & 0 & 0 & 0 & 0 & 0 \\ 0 & 0 & \frac{T_{12} T_{32}}{T_{12}^{2}+T_{22}^{2}} & \frac{-T_{22} T_{32}}{T_{12}^{2}+T_{22}^{2}} & 1 & 0 & 0 & 0 & 0 & 0\end{array}\right]$

The observation noise, $V=\left[\begin{array}{lllll}V_{v x} & V_{v y} & V_{\varphi x} & V_{\varphi y} & V_{\varphi z}\end{array}\right]^{T}$

During the simulation, each element can be regarded as zero mean white noise.

Calman Filtering Algorithm. We use the method of discretization to process the state equation and measurement equation.

$$
X_{k}=\Phi_{k, k-1} X_{k-1}+\Gamma_{k-1} W_{k-1}, Z_{k}=H_{k} X_{k}+V_{k}
$$

In the above formula, ${ }^{X_{k}}$ represents the system state vector, $\Phi_{k, k-1}$ represents the system transfer matrix, ${ }^{\Gamma_{k-1}}$ represents the system noise matrix, ${ }^{W_{k-1}}$ represents a discrete system white noise with zero mean, $Z_{k}$ is a measurement vector, $H_{k}$ is a measurement matrix, $V_{k}$ is a discrete zero mean white measurement noise vector. $X_{k}, W_{k-1}, V_{k}$, they are not related.

Put the following basic equation to represent the discretization Calman filter[8].

The state estimation equation of prediction is as follows. $\hat{X}_{k / k-1}=\Phi_{k, k-1} \hat{X}_{k-1}$

Variance prediction equation is as follows. $P_{k / k-1}=\Phi_{k, k-1} P_{k-1} \Phi_{k, k-1}^{T}+\Gamma_{k-1} Q_{k-1} \Gamma_{k-1}^{T}$

State estimate update equation is as follows. $\hat{X}_{k}=\hat{X}_{k / k-1}+K_{k}\left(Z_{k}-H_{k} \hat{X}_{k / k-1}\right)$

Variance iterative update equation is as follows. $P_{k}=\left(I-K_{k} H_{k}\right) P_{k / k-1}\left(I-K_{k} H_{k}\right)^{T}+K_{k} R_{k} K_{k}^{T}$

The filter gain renewal equation is as follows. $K_{k}=P_{k / k-1} H_{k}^{T}\left(H_{k} P_{k / k-1} H_{k}^{T}+R_{k}\right)^{-1}$

According to above equation, Calman filter corresponding to update process is as follows in Fig. 2. 


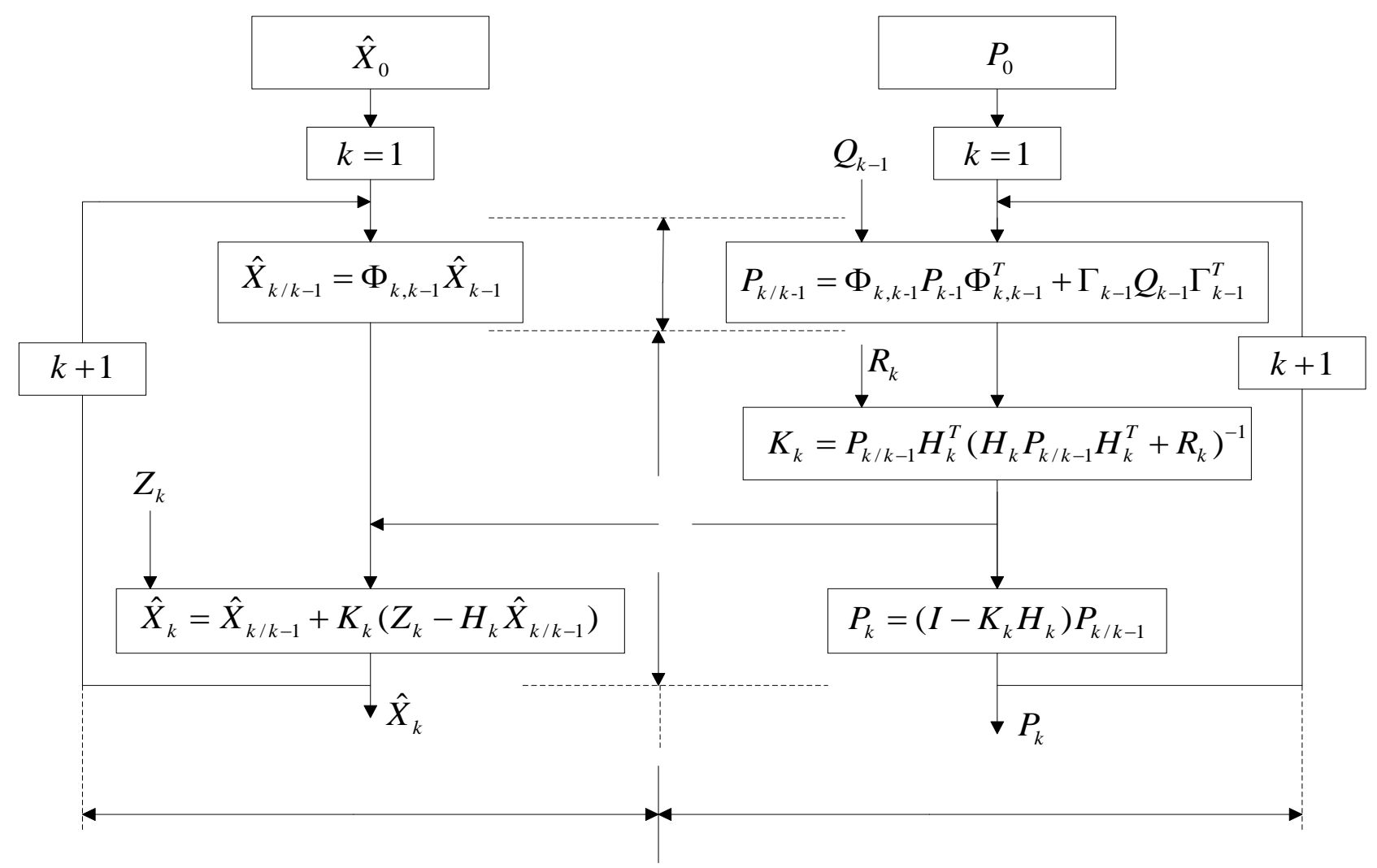

Fig. 2 The realization process of a discrete Calman filter

\section{Simulation Analysis}

The variation of the attitude angle of photoelectric aiming equipment relative navigation, which is caused by the carrier moving, this is the main factor affecting the precision of stable targeting. The main inertial reference information is baseline information. The platform misalignment angle, which is a sub inertial relative principal ins, is the main error source of impacting stable targetin.

When the chariot driving in mean velocity on a bumpy road, trajectory generator generates the information of gyroscope and accelerometer. The initial geographical longitude of the carrier is $116.3436^{\circ}$, latitude is $39.9775^{\circ}$. In MINS gyroscope,the zero drift of gyroscope is $0.01^{\circ} / \mathrm{h}$, random drift is $0.005^{\circ} / \mathrm{h}$. The accelerometer zero drift is $10 \mu \mathrm{g}$, random bias is $5 \mu \mathrm{g}$. In SINS gyroscope, the zero drift of gyroscope is $0.1^{\circ} / \mathrm{h}$, random drift is $0.05^{\circ} / \mathrm{h}$. The accelerometer zero drift is $100 \mu \mathrm{g}$, random bias is $50 \mu \mathrm{g}$.The initial values of three attitude angles respectively are $0^{\circ}, 0^{\circ}, 30^{\circ}$. Their change process are as sine wave, but the initial amplitude, period, and phase are different.Fixed attitude angle, which is A relative to $\mathrm{B}$, are respectively for the following numerical: $\psi_{x}$ is $25^{\prime}, \psi_{y}$ is $30^{\prime}, \psi_{z}$ is $45^{\prime}$. The chariots running speed is $50 \mathrm{~km} / \mathrm{h}$, the navigation period is $0.1 \mathrm{~s}$, total simulation time is 120s. The method of transfer alignment is based on velocity plus attitude matching algorithm. Through the Kalman filter, we estimated the platform misalignment angle,which is SINS relative to MINS. The results obtained is shown in Fig. 3

As can be seen from the figure, in the case of additive noise, the method of transfer alignment is based on velocity plus attitude matching can quickly estimate the SINS platform misalignment angle. The alignment time is less than 9s. The East, North and Up three direction misalignment angle are respectively in the 25 ', 30', 45 'near the change. The amplitude of variation is within the range of 3 ' , and the angle value is basically the same as the actual value. 

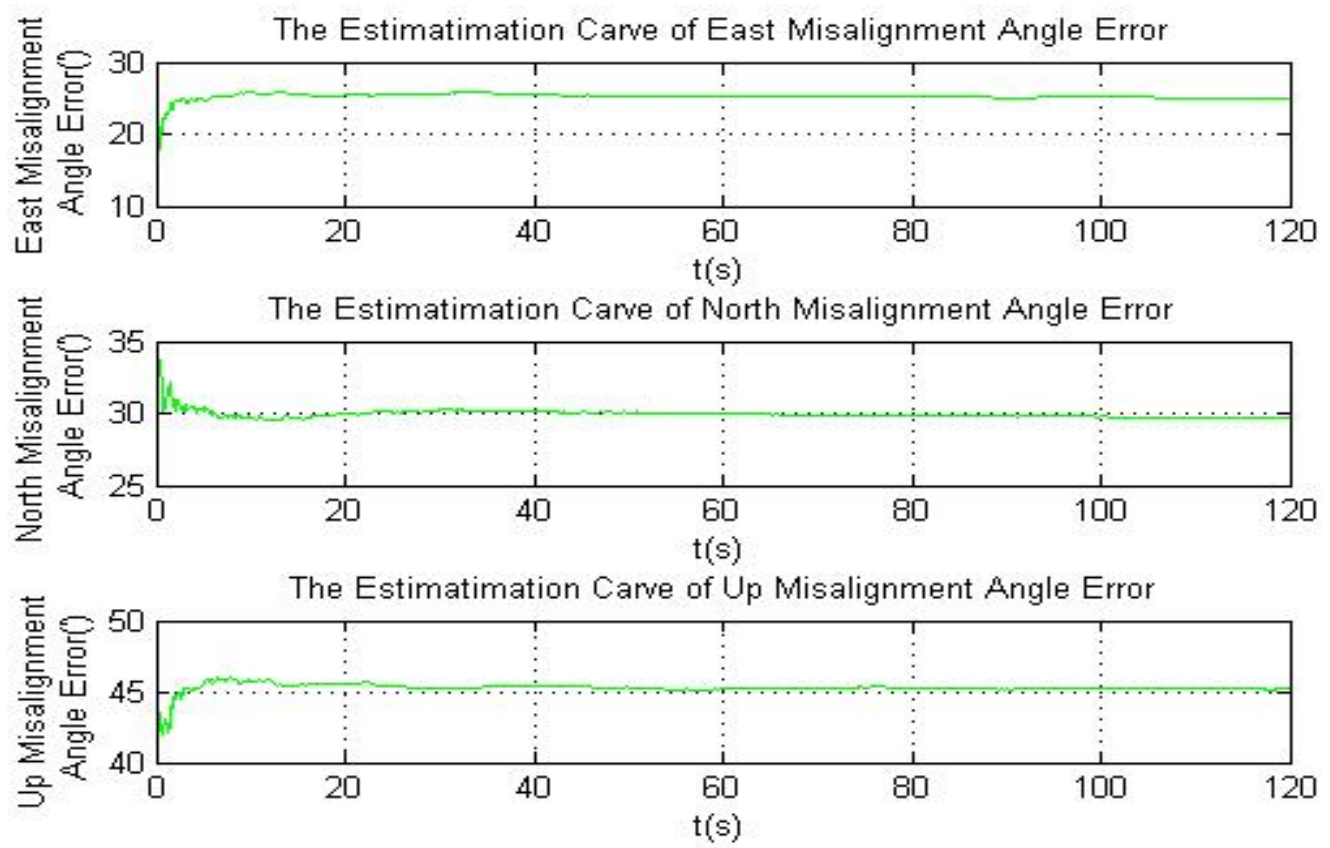

Fig. 3 The misalignment angle estimation value of SINS relative to MINS

The navigation coordinate system obtained by single IMU measurement solution exists the platform misalignment angles, compared to the true navigation system. Method can quickly estimate and compensate teh SINS platform misalignment angles, reduce the attitude calculation error, to improve the precision of stable targeting.

\section{Conclusions}

Due to single IMU stabilized targeting system cannot achieve rapid initial alignment and attitude measurement accuracy is influenced by zero drift, we propose transfer alignment stable targeting method based on velocity plus attitude matching algorithm. The simulation results show that, this method can quickly, accurately estimate misalignment angles of photoelectric equipment sub inertial navigation platform, the misalignment angle could compensate angle attitude of photoelectric equipment. On the basis of the original, we greatly improved the precision of stable targeting. The effect of fast and accurate alignment is well, it can effectively improve missile combat effectiveness.

\section{References}

[1] Charya A, Sadhu S, Ghoshal T K:Aerospace Science and Technology Mater Vol.125-128 (2011),

[2] Yongyuan Qin: Inertial navigation (Science Press, Beijing 2006),In Chinese.

[3] Xiaolin Gong, Journal of Beihang University, 2008, 34(8): 865-868,In Chinese.

[4] Zhou Naixin, Wang Si,Proceed of the 25th Chinese Control Conference.Harbin:2006:27-31.

[5] LYOU J,LIM Y C. Aeronautical and Space Sciences， 2009， 48( 61) : 143-151.

[6] Yinghong Han,Wanchun Chen. Journal of Beihang University, 2012, 38(2): 149-152,In Chinese.

[7] Yunjun,Hao Lu, Journal of missiles and guidance, 2012, 32(1):21-26,In Chinese.

[8] Saicheng Wu. Chinese Journal of scientific instrument, 2011,32(1):201-205. 